\title{
Completeness of Pledger's modal logics of one-sorted projective and elliptic planes
}

\author{
Robert Goldblatt \\ School of Mathematics and Statistics \\ Victoria University of Wellington
}

\begin{abstract}
Ken Pledger devised a one-sorted approach to the incidence relation of plane geometries, using structures that also support models of propositional modal logic. He introduced a modal system $12 \mathrm{~g}$ that is valid in one-sorted projective planes, proved that it has finitely many non-equivalent modalities, and identified all possible modality patterns of its extensions. One of these extensions $8 \mathrm{f}$ is valid in elliptic planes. These results were presented in his 1980 doctoral dissertation [14], which is reprinted in this issue of the Australasian Journal of Logic.

Here we show that $12 \mathrm{~g}$ and $8 \mathrm{f}$ are strongly complete for validity in their intended one-sorted geometrical interpretations, and have the finite model property. The proofs apply standard technology of modal logic (canonical models, filtrations) together with a step-by-step procedure introduced by Yde Venema for constructing two-sorted projective planes.
\end{abstract}

AMS 2020 classification $\quad$ 03B45, 51A05, 15M10

Keywords modal logic, projective plane, elliptic plane, strongly complete, finite model property, filtration

\section{Pledger's dissertation}

Kenneth Ernest Pledger (1938-2016) was a mathematician who taught at the Victoria University of Wellington for 52 years. He was primarily interested in geometry and the history of mathematics, but participated regularly in logic seminars conducted jointly by mathematicians and philosophers at VUW. This led him to make some contributions to modal logic, including the articles [11, 12, 13]. In the mid1970 's he independently discovered the fact, of significance to provability logic, 
that any normal modal logic containing the Löb axiom $\square(\square \varphi \rightarrow \varphi) \rightarrow \square \varphi$ must extend the logic K4, i.e. must contain the transitivity axiom $\square \varphi \rightarrow \square \square \varphi$ (see [4, p. 11]). He also showed that any normal modal logic containing the Grzegorczyk axiom $\square(\square(\varphi \rightarrow \square \varphi) \rightarrow \varphi) \rightarrow \varphi$ is an extension of S4 [4, pp. 157, 259].

In 1981 Pledger was awarded the PhD degree by the University of Warsaw for a dissertation [14] entitled Some interrelations between geometry and modal logic, with Lesław Szczerba (1938-2010) as promoter. Their relationship had developed from a sabbatical visit made by Szczerba in 1977 to Wellington, followed by one made by Pledger in 1979 to Warsaw, where the University's regulations for doctoral study did not include a residence requirement. Szczerba was a former student of Wanda Szmielew. He worked mainly on the foundations of geometry, including in collaboration with Tarski [16].

Pledger's dissertation set out a one-sorted approach to plane geometry. The traditional approach to this subject is based on two-sorted structures whose sorts are a set $P$ of points and a set $L$ of lines, with a binary incidence relation $I$ from $P$ to $L$. When $a I \ell$ the point $a$ is incident with the line $\ell$. If we allow this to also be written $\ell I a$, then $I$ is extended to a symmetric relation on $P \cup L$. Then as $a I \ell I a$ we get that $a I^{2} a$, where $I^{2}$ is the relational composition of $I$ with itself. In a projective plane, any two points $a, b$ have a line $\ell$ passing through both, so $a I \ell I b$ and $a I^{2} b$. Each point is $I^{2}$-related to all points and not to any lines, and dually each line is $I^{2}$-related to all lines and not to any points.

The central idea of [14] was that the two-sorted theory "can be made onesorted by keeping careful account of whether the incidence relation is iterated an even or odd number of times" [14, p.1]. This was implemented by imposing firstorder conditions on an abstract structure $(X, I)$ with $I \subseteq X \times X$ that ensure that the composition $I^{2}$ is an equivalence relation on the set $X$ that has at most two equivalence classes. When it has exactly two, one of them can be chosen as $P$ and the other as $L$ to obtain the structure of a two-sorted projective plane. When there is only one $I^{2}$-equivalence class, it exhibits the structure of an elliptic plane, a type of geometry that was classically modelled by the surface of a Euclidean sphere with antipodal points identified.

We will refer to the structures $(X, I)$ defined in [14] for which this analysis holds as one-sorted planes. Those with two $I^{2}$-equivalence classes are called projective in [14]. They include the structures $(P \cup L, I)$ where $I$ is the symmetric incidence relation of a traditional two-sorted projective plane based on $P$ and $L$. The ones with a single $I^{2}$-equivalance class are called elliptic.

Now a structure of the form $\mathscr{F}=(X, I)$ is known in modal logic as a Kripke frame, although Kripke called it a model structure [9]. A model on $\mathscr{F}$ for the language of modal logic is given by a truth relation specifying which formulas are true at which points of $X$, with $\square \varphi$ being true at point $a$ when $\varphi$ is true at all points 
of $\{b \in X: a I b\}$. A formula is valid in the frame $\mathscr{F}$ if it is true at every point in every model on $\mathscr{F}$.

Some of the first-order conditions defining a plane $\mathscr{F}$ are equivalent to the validity in $\mathscr{F}$ of certain modal formulas. This led Pledger to study a logic, which he called $12 \mathrm{~g}$, which can be described as the extension of the modal logic KDB by the axiom

$$
\square \square \varphi \rightarrow \square \square \square \square \varphi
$$

whose validity is equivalent to $I^{2}$ being transitive. Here KDB is the smallest normal modal logic to contain the deontic axiom

$$
\mathrm{D}: \quad \square \varphi \rightarrow \diamond \varphi
$$

which corresponds to $I$ being serial (each element is $I$-related to something), and the Brouwerian axiom

$$
\mathrm{B}: \diamond \square \varphi \rightarrow \varphi
$$

which corresponds to symmetry of $I .12 \mathrm{~g}$ is sound for validity in all one-sorted planes, including those derived from two-sorted projective planes as well as the elliptic ones.

The name ' $12 \mathrm{~g}$ ' relates to the fact that this logic has 12 non-equivalent proper affirmative modalities [14, (37)], these being the finite sequences of the symbols $\square$ and $\diamond 1$ Any such sequence is reducible to one of 12 cases. It is shown in [14, pp.12-15] that there are nine different logics that arise by adding to $12 \mathrm{~g}$ an axiom of the form $A \varphi \rightarrow B \varphi$, where $A$ and $B$ are affirmative modalities. One of these logics, called $8 \mathrm{f}$, can be obtained by adding to $12 \mathrm{~g}$ the axiom

$$
\mathrm{T}^{3}: \quad \square \square \square \varphi \rightarrow \varphi
$$

(the reason for the name $\mathrm{T}^{3}$ will be given in Section 6 ).

$8 \mathrm{f}$ is sound for validity in one-sorted elliptic planes. This can be illustrated in the classical model of the real elliptic plane, whose lines correspond to the great circles on a Euclidean sphere (with antipodal points identified). Each point has an associated line called its polar: if the point is taken as the north pole then its polar is the equator. Each line is the polar of a unique point, called the pole of the line. The correspondence between poles and polars gives a bijection between the set of points and the set of lines. Representing each line by its pole allows the geometry to be determined by one sort of object (points), using the binary relation $a I b$ that

\footnotetext{
${ }^{1}$ The naming system used in [14] labels each logic by the number of its proper affirmative modalities and a distinguishing letter.
} 
holds between points $a$ and $b$ when $b$ lies on the polar of $a$, which implies that $a$ lies on the polar of $b$. In this situation $a$ and $b$ are said to be conjugate. If $c$ is the pole of the line joining two points $a$ and $b$, then $a I c I b$, showing that $a I^{2} b$. Hence there is one $I^{2}$-equivalence class. Raphael Robinson[15] showed that the conjugacy relation can serve as the sole primitive from which to construct the geometry of the real elliptic plane. The line corresponding to a point $a$ is recoverable as the set $\{b: a I b\}$ of points conjugate to $a$. Marek Kordos [8] has given an axiomatisation of plane elliptic geometry over formally real Pythagorean fields as a theory of a single binary relation.

Now a feature of the real elliptic plane is the notion of a self-polar triangle, a triple $a, b, c$ of points such that each is the pole of the line joining the other two, implying that $a I b I c I a$. Hence $a I^{3} a$ where $I^{3}$ is the 3 -fold composition of $I$ with itself. Each point $a$ is a vertex of a self-polar triangle so $I^{3}$ is reflexive, a property that is equivalent to the validity of $\mathrm{T}^{3}$. In Pledger's theory, reflexivity of $I^{3}$ ensures that a one-sorted plane $(X, I)$ has only one $I^{2}$-equivalence class and so is elliptic in that sense.

Some of the first-order conditions defining a plane are not equivalent to the validity of any modal formula. This was shown in Chapter VII of [14] by exhibiting counter-examples in which the condition in question fails to be preserved by a surjective bounded morphism, a type of map between frames that preserves validity of modal formulas. Most notably this failure applies to the property of uniqueness of the line joining two given points, and of the point at which two lines meet.

At the end of [14] the problem is raised of axiomatising the logic comprising all the modal formulas that are valid in all one-sorted projective and elliptic planes. As mentioned above this logic includes the system $12 \mathrm{~g}$, since $12 \mathrm{~g}$ is sound for validity in this class of planes. We will prove here that $12 \mathrm{~g}$ is also complete for validity in this class: any formula valid in these planes is a theorem of $12 \mathrm{~g}$, so the sought for logic is just $12 \mathrm{~g}$ itself. The proof makes use of a construction developed by Yde Venema [17] a couple of decades after Pledger's work.

In the 1990's a number of modal logicians became interested in the use of incidence geometries as models of various modal languages (see the references of [17] and the review article [2] for information about this). Venema's contribution was to axiomatise the logic of two-sorted projective planes in a two-sorted modal language. In so doing he gave a step-by-step procedure for constructing a two-sorted projective plane as a bounded morphic preimage of a quasi-plane, a structure satisfying the existence properties of projective planes, but not their uniqueness properties.

Now the completeness of $12 \mathrm{~g}$ can also be expressed by saying that every $12 \mathrm{~g}$ consistent formula is satisfiable, i.e. true at some point, in a model on a projective or elliptic plane. We will prove the stronger property that every $12 \mathrm{~g}$-consistent set 
of formulas is satisfiable in a model on the one-sorted plane $(P \cup L, I)$ derived from some two-sorted projective plane. We define a one-sorted notion of quasi-plane, and show that if $\mathscr{F}$ is one of these and is connected, then it is a bounded morphic image of a two-sorted quasi-plane (Theorem 5). Combined with the construction from [17], we then infer that $\mathscr{F}$ is a bounded morphic image of the one-sorted plane derived from a two-sorted projective plane (Corollary 6 .

We then go on to show that $8 \mathrm{f}$ axiomatises the modal logic of elliptic one-sorted planes. The construction from [17] does not seem applicable for this, since it leads to projective planes. Instead we apply the step by step method of [17] to directly construct a one-sorted elliptic plane as a bounded morphic preimage of a one-sorted quasi-plane (Theorem 8).

In the final section, the filtration method is used to show that $12 \mathrm{f}$ and $8 \mathrm{~g}$ have the finite model property, i.e. are characterised by validity in finite structures, which implies that they are decidable.

\section{2-planes}

A two-sorted frame, or 2-frame, is a structure $\mathscr{F}=(P, L, I)$ with $P \cap L=\emptyset$ and $I \subseteq P \times L$. Elements of $P$ and $L$ are called points and lines, respectively, and $I$ is the incidence relation. When $a I b$ we may say that $a$ and $b$ are incident, that $a$ lies on $b$, that $b$ passes through $a$, etc. Given points are said to be collinear if there exists a line passing through all of them. A quadrangle is a sequence of four points, no three of which are collinear. $\mathscr{F}$ is a projective plane if it satisfies the following axioms:

P1: any two distinct points have exactly one line passing through them both.

P2: any two distinct lines have exactly one point lying on them both.

P3: there exists a quadrangle.

Here (P3) is a non-degeneracy condition. It is equivalent to requiring that the plane contains a triangle (three non-collinear points) and every line has at least three points. We sometimes call a projective plane a projective 2-plane to emphasise the number of sorts involved.

A quasi-plane [17, Def. 3.1] is a 2-frame satisfying:

Q1: any two points have at least one line passing through them both.

Q2: any two lines have at least one point lying on them both. 
The two points and two lines hypothesized in these statements are not required to be distinct. Thus in a quasi-plane, any point is incident with at least one line, and any line with at least one point.

If $\mathscr{F}=(P, L, I)$ and $\mathscr{F}^{\prime}=\left(P^{\prime}, L^{\prime}, I^{\prime}\right)$ are 2-frames, then a homomorphism from $\mathscr{F}$ to $\mathscr{F}^{\prime}$ is given by a function $\theta: P \cup L \rightarrow P^{\prime} \cup L^{\prime}$ with $\theta(P) \subseteq P^{\prime}$ and $\theta(L) \subseteq L$ ' that satisfies the 'Forth' condition

F: $a I b$ implies $\theta(a) I^{\prime} \theta(b)$.

A homomorphism $f$ is a bounded morphism if the following 'Back' conditions hold for any $a \in P$ and $b^{\prime} \in L^{\prime}$ in $\mathrm{B} 1$, and any $a^{\prime} \in P^{\prime}$ and $b \in L$ in B2.

B1: If $\theta(a) I^{\prime} b^{\prime}$, then there exists $b$ such that $a I b$ and $\theta(b)=b^{\prime}$.

B2: If $a^{\prime} I^{\prime} \theta(b)$, then there exists $a$ such that $a I b$ and $\theta(a)=a^{\prime}$.

If there exists a bounded morphism from $\mathscr{F}$ to $\mathscr{F}^{\prime}$ that is surjective, then we say that $\mathscr{F}^{\prime}$ is a bounded morphic image of $\mathscr{F}^{\prime}$.

Theorem 1. [17, Theorem 3.2] Every quasi-plane is a bounded morphic image of a projective plane.

Venema's technique for proving this result starts with a quasi-plane $\mathscr{F}^{\prime}$ and a diagram consisting of a point $a$ and a line $b$ of $\mathscr{F}^{\prime}$ that are incident and, in a step by step manner, adds points and lines to the diagram in order to fulfil the existential assertions made by P3, Q1, Q2, B1 and B2. This is done in such a way that at each step the diagram constructed so far has a homomorphism to $\mathscr{F}^{\prime}$ and preserves the uniqueness requirements of $\mathrm{P} 1$ and $\mathrm{P} 2$. At the end of the construction, the diagram has become a projective plane with a bounded morphism onto $\mathscr{F}^{\prime}$.

The technique was applied to affine planes by Hodkinson and Hussain in [7]. In Section 4 we will apply it to one-sorted structures.

\section{1-planes}

A one-sorted frame, or 1-frame is a structure $\mathscr{F}=(X, I)$ with $I \subseteq X \times X$. We continue to refer to $I$ as an incidence relation. $I$ is serial if its domain is $X$, i.e. every element $a$ of $X$ is incident $(a I b)$ with some element $b$. We sometimes attribute a property of $I$ to $\mathscr{F}$, saying that $\mathscr{F}$ is serial when $I$ is etc.

Viewing $\mathscr{F}$ as a graph, we say that a sequence in $\mathscr{F}$ of the form $a_{0} I a_{1} I \cdots I a_{n}$ is an I-path from $a_{0}$ to $a_{n}$ of length $n \geq 1$ (the $a_{i}$ 's need not be distinct). Let $I^{n}$ be the $n$-fold relational composition of $I$ with itself. Then $a I^{n} b$ iff there exists an $I$-path of length $n$ in $\mathscr{F}$ from $a$ to $b$. We also let $I^{0}$ be the identity relation on $X$. 
A 4-cycle is a path aIbIcIdIa of length 4 from an element to itself. It is proper if $a \neq c$ and $b \neq d$.

A one-sorted plane, or 1-plane, is a 1-frame that satisfies the three conditions

O1: For all $a$ and $b, a I^{4} b$ implies $a I^{2} b$.

O2: For all $a$ and $b, a I^{2} b$ or $a I^{3} b$.

O3: There are no proper 4-cycles, i.e. aIbIcIdIa implies $a=c$ or $b=d$.

It is called non-degenerate if it also satisfies

O4: There exist $a, b, c, d, e, f$ with aIbIcId and $e I f$, but $a$ is not incident with $d$, and none of $a, b, c, d$ is incident with $e$ or $f$.

Let $I_{+}=I \cup\{(b, a): a I b\}$, the smallest symmetric relation including $I$. I itself is symmetric iff $I_{+}=I$. If $\mathscr{F}=(P, L, I)$ is a 2 -frame, let $\mathscr{F}_{+}$be the 1 -frame $\left(P \cup L, I_{+}\right)$.

Theorem 2. [14, Chapter I] If $\mathscr{F}$ is a projective 2-plane, then $\mathscr{F}_{+}$is a non-degenerate 1-plane in which $P$ and $L$ are distinct equivalence classes under $I_{+}^{2}$, and the only such classes.

Proof. Conditions O1-O4 are axioms (1)-(4) of [14]. We reprise the arguments given there for the validity in $\mathscr{F}_{+}$of these conditions. For O1, if $a I_{+}{ }^{n} b$ with $n$ even, then $a$ and $b$ are both points or both lines in $\mathscr{F}$, hence $a I_{+}{ }^{2} b$ as noted in Section 1

For $\mathrm{O} 2$, if $a$ and $b$ are of the same sort in $\mathscr{F}$, then $a I_{+}{ }^{2} b$ as just observed. If $a$ is a point and $b$ is a line, take any line $c$ through $a$ and let point $d$ lie on $b$ and $c$ : then $a I_{+} c I_{+} d I_{+} b$, hence $a I_{+}{ }^{3} b$. If $b$ is a point and $a$ is a line, then $b I_{+}{ }^{3} a$ by the previous sentence, hence $a I_{+}{ }^{3} b$.

$\mathrm{O} 3$ captures the uniqueness expressed in $\mathrm{P} 1$ and $\mathrm{P} 2$. Let $a I_{+} b I_{+} c I_{+} d I_{+} a$. If $a$ is a point, then so is $c$ while $b$ and $d$ are lines passing through both points, hence if $a \neq c$ then $b=d$ by P1. If $a$ is a line, then so is $c$ while $b$ and $d$ are points on both lines, so again if $a \neq c$ then $b=d$ by $\mathrm{P} 2$.

$\mathrm{O} 4$ captures the non-degeneracy expressed by P3, which ensures that $\mathscr{F}$ has some quadrangle $b, d, e, g$. Figure 1 shows how to then obtain lines $a, c, f$ so that $a, b, c, d, e, f$ fulfils $\mathrm{O}_{4}$ in $\mathscr{F}_{+}$[14, Figure 6].

That proves $\mathscr{F}_{+}$is a non-degenerate 1-plane. Now given distinct points $a, b \in P$, there is a line $c \in L$ passing through both, hence $a I_{+} c I_{+} b$ so $a I_{+}^{2} b$. Also any point $a$ is incident with some line $c$, so that $a I_{+} c I_{+} a$ and hence $a I_{+}^{2} a$. Thus any point is $I^{2}$-related to all points, but not to any lines because $I$ only connects elements of different type, so $I^{2}$ only connects elements of the same type. Dually, any line is $I^{2}$-related to all lines, but not to any points. Since $P$ and $L$ are disjoint they form a partition of $P \cup L$ with each being an $I^{2}$-equivalence class. 


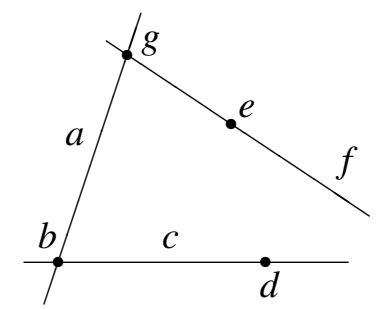

Figure 1: non-degeneracy

A 1 -frame $\mathscr{F}=(X, I)$ is connected if any two of its elements have an $I_{+}$-path from one to the other. If $I$ is symmetric, this is an $I$-path. It follows from $\mathrm{O} 2$ that a 1-plane is connected.

It was shown in [14, Chapter 1] that in any 1-plane, $I$ is serial and symmetric, $I^{2}$ is an equivalence relation with at most two equivalence classes, and when there are two such classes then by taking one as $P$ and the other as $L$ and restricting $I$ to $P \times L$ we obtain a 2-frame satisfying P1and P2. If the 1-plane is non-degenerate, then P3 holds as well, so it is a projective plane. When there is one $I^{2}$-equivalence class, by regarding each element as both a point and a line we can reach the same conclusion.

It was then observed on [14, p.7] that parts of this analysis depend only on weaker assumptions. To explain this we define a quasi-1-plane to be any 1-frame that is serial and symmetric and satisfies O1. Thus any 1-plane is a quasi-1-plane. The quasi-planes of the previous section may be called quasi-2-planes to distinguish them from the one-sorted notion just defined.

Theorem 3. Let $\mathscr{F}=(X, I)$ be a quasi-1-plane. Then:

(1) $I^{2}$ is an equivalence relation.

(2) aIb implies $a I^{3} b$.

(3) $\mathscr{F}$ is connected iff it satisfies $O 2$.

(4) If $\mathscr{F}$ is connected, then for any element a, the subsets $I^{2}(a)=\left\{x: a I^{2} x\right\}$ and $I^{3}(a)=\left\{x: a I^{3} x\right\}$ are the only $I^{2}$-equivalence classes.

(5) If $\mathscr{F}$ is connected, then for any element $a$, if $x I y$ then one of $x$ and $y$ is in $I^{2}(a)$ and the other is in $I^{3}(a)$.

Proof. (1) Reflexivity of $I^{2}$ : for any $a$ there a $b$ with $a I b$ by seriality of $I$, so then $a I b I a$ by symmetry of $I$, hence $a I^{2} a$. Symmetry of $I^{2}$ follows from symmetry of $I$. Transitivity of $I^{2}$ is just what $\mathrm{O} 1$ asserts. 
(2) If $a I b$, then $a I b I^{2} b$ by (1), giving $a I^{3} b$.

(3) $\mathrm{O} 2$ implies that any two points have an $I$-path between them of length 2 or 3 , so $\mathscr{F}$ is connected. For the converse, take $a, b \in X$. If $a=b$, then $a I^{2} b$ by (1). If $a \neq b$, then assuming $\mathscr{F}$ is connected, since $I$ is symmetric we have $a I^{n} b$ for some $n \geq 1$. If $n=1$ then $a I^{3} b$ by (2). If $n \geq 4$, then since O1 ensures that any path of length 4 between two points can be replaced by one of length 2 , we get that $a I^{n-2} b$, then $a I^{n-4} b$ etc., leading to either $a I^{2} b$ or $a I^{3} b$. Hence $\mathrm{O} 2$ holds.

(4) (See [14, proof of (13)].) Assume $\mathscr{F}$ is connected, and hence satisfies $\mathrm{O} 2$ by (3). Fix any $a \in X$. As in (1), there is some $b$ with $a I b I a$. If $a I^{3} x$, then $b I a I^{3} x$, so $b I^{4} x$ and hence $b I^{2} x$ by O1. Conversely, if $b I^{2} x$ then $a I b I^{2} x$ and so $a I^{3} x$. This shows that $I^{3}(a)$ is the $I^{2}$-equivalence class $I^{2}(b)$ of $b$. But by O2, for any $x$, either $a I^{2} x$ or $a I^{3} x$, so the $I^{2}$-equivalence classes of $a$ and $b$ are all that there are.

(5) Let $x I y$. If $x \in I^{2}(a)$, then $a I^{2} x I y$, so $y \in I^{3}(a)$. But if $x \notin I^{2}(a)$, then $x \in I^{3}(a)$ by part (4), so $a I^{3} x I y$, hence $a I^{4} y$, then $y \in I^{2}(a)$ by O1.

By part (4) of this result, all connected quasi-1-planes, which includes all the 1planes, have at most two $I^{2}$-equivalence classes. Those with two classes will called projective quasi-1-planes, while those with one class are elliptic. So Theorem 2 states that if $\mathscr{F}$ is a projective 2 -plane, then $\mathscr{F}_{+}$is a non-degenerate projective 1-plane.

Now a symmetric 1-frame can be viewed as an undirected graph with an edge joining vertices $a$ and $b$ just when $a I b$ (hence $b I a$ ). Figure 2 shows two such
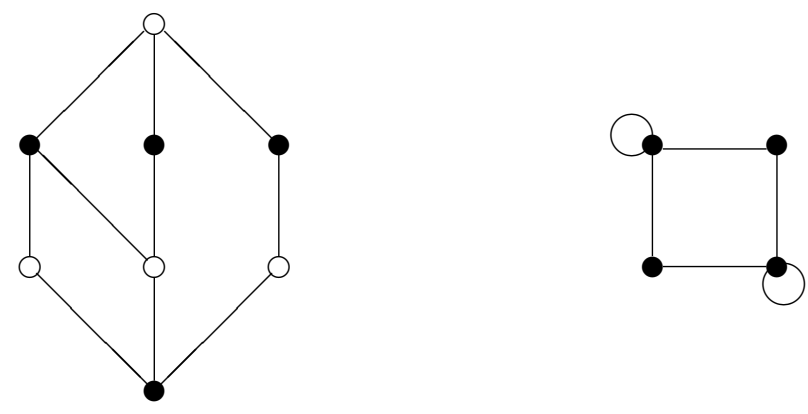

Figure 2: two quasi-1-planes, not 1-planes

graphs, which are Figures 105 and 107 from [14]. The left one is a projective quasi-1-plane with the black and white circles displaying the two $I^{2}$-equivalence 
classes. This graph also satisfies the non-degeneracy condition $\mathrm{O} 4$. The right graph is an elliptic quasi-1-plane, with the two looped edges displaying $I$-reflexive vertices. Both graphs contain proper 4-cycles violating O3, so neither is a 1-plane.

We now extend the notion of bounded morphism to variously-sorted structures. First, a bounded morphism from a 1-frame $\mathscr{F}=(X, I)$ to a 1-frame $\mathscr{F}^{\prime}=\left(X^{\prime}, I^{\prime}\right)$ is given by a function $\theta: X \rightarrow X^{\prime}$ that is a homomorphism, i.e. satisfies the forth condition $\mathrm{F}$ for all $a, b \in X$, and satisfies the back condition B1 for all $a \in X$ and $b \in X^{\prime}$. This is the standard notion of bounded morphism for 1-frames in modal logic. It is a routine fact that the functional composition of two bounded morphisms of this type is also a bounded morphism.

Next we define a bounded morphism from a 2-frame $\mathscr{F}=(P, L, I)$ to a 1-frame $\mathscr{F}^{\prime}=\left(X^{\prime}, I^{\prime}\right)$ as being given by a function $\theta: P \cup L \rightarrow X^{\prime}$ that satisfies the homomorphism condition F for all $a \in P$ and $b \in L$, the condition B1 for all $a \in P$ and $b \in X^{\prime}$; and the condition $\mathrm{B} 2$ for all $a^{\prime} \in X^{\prime}$ and $b \in L$.

Lemma 4. (1) If $\theta$ is a bounded morphism from a 2-frame $\mathscr{F}=(P, L, I)$ to a 2-frame $\mathscr{F}^{\prime}=\left(P^{\prime}, L^{\prime}, I^{\prime}\right)$, and $\tau$ a bounded morphism from $\mathscr{F}^{\prime}$ to a 1-frame $\mathscr{F}^{\prime \prime}=\left(X^{\prime \prime}, I^{\prime \prime}\right)$, then the composition $\tau \circ \theta$ is a bounded morphism from $\mathscr{F}$ to $\mathscr{F}^{\prime \prime}$.

(2) If $\theta$ is a bounded morphism from a 2-frame $\mathscr{F}=(P, L, I)$ to a 1-frame $\mathscr{F}^{\prime}=$ $\left(X^{\prime}, I^{\prime}\right)$, and $I^{\prime}$ is symmetric, then $\theta$ is also a bounded morphism from the 1-frame $\mathscr{F}_{+}=\left(P \cup L, I_{+}\right)$to $\mathscr{F}^{\prime}$.

Proof. (1) $\tau \circ \theta$ is a homomorphism because $\theta$ and $\tau$ are: if $a I b$ then $\theta(a) I^{\prime} \theta(b)$ by $\mathrm{F}$ for $\theta$ and so by $\mathrm{F}$ for $\tau, \tau(\theta(a)) I^{\prime \prime} \tau(\theta(b))$, i.e. $(\tau \circ \theta)(a) I^{\prime \prime}(\tau \circ \theta)(b)$.

For B1, if $\tau(\theta(a)) I^{\prime \prime} b^{\prime \prime}$, then by B1 for $\tau$ there exists $b^{\prime} \in L^{\prime}$ with $\theta(a) I^{\prime} b^{\prime}$ and $\tau\left(b^{\prime}\right)=b^{\prime \prime}$. Hence by B1 for $\theta$, there exists $b \in L$ with $a I b$ and $\theta(b)=b^{\prime}$, hence $(\tau \circ \theta)(b)=b^{\prime \prime}$. The proof of B2 for $\tau \circ \theta$ is similar,

(2) F: let $a I_{+} b$ in $\mathscr{F}_{+}$. If $a I b$, then $\theta(a) I^{\prime} \theta(b)$ by $\mathrm{F}$ for $\theta: \mathscr{F} \rightarrow \mathscr{F}^{\prime}$. Otherwise we have $b I a$, hence $\theta(b) I^{\prime} \theta(a)$ likewise, and so again $\theta(a) I^{\prime} \theta(b)$ as $I^{\prime}$ is symmetric. This shows that $\mathrm{F}$ holds for $\theta: \mathscr{F}_{+} \rightarrow \mathscr{F}^{\prime}$.

B1: let $\theta(a) I^{\prime} b^{\prime}$ in $\mathscr{F}^{\prime}$. If $a \in P$, then by B1 for $\theta: \mathscr{F} \rightarrow \mathscr{F}^{\prime}$ there exists $b \in L$ with $a I b$, hence $a I_{+} b$, and $\theta(b)=b^{\prime}$. Otherwise we have $a \in L$. But $b^{\prime} I^{\prime} \theta(a)$ by symmetry, so by B2 for $\theta: \mathscr{F} \rightarrow \mathscr{F}^{\prime}$ there exists $b \in P$ with $b I a$, hence $a I_{+} b$, and $\theta(b)=b^{\prime}$. This shows B1 holds for $\theta: \mathscr{F}_{+} \rightarrow \mathscr{F}^{\prime}$.

Theorem 5. Every connected quasi-1-plane is a bounded morphic image of a quasi-2-plane. 
Proof. Let $\mathscr{F}=(X, I)$ be a connected quasi-1-plane. By Theorem 3 4$), \mathscr{F}$ has at most two $I^{2}$-equivalence classes. Take first the case that it has exactly two. Take an element $a$ and let $P=\left\{x: a I^{2} x\right\}$ and $L=\left\{x: a I^{3} x\right\}$. Theorem 3(4) says these are the two $I^{2}$-equivalence classes, so they are disjoint and partition $X$. Form the 2-frame $\mathscr{F}^{\prime}=\left(P, L, I^{\prime}\right)$, where $I^{\prime}$ is the restriction of $I$ to $P \times L$. Let $\theta: P \cup L \rightarrow X$ be the identity function.

Since $I^{\prime} \subseteq I, \theta$ is a homomorphism from $\mathscr{F}^{\prime}$ onto $\mathscr{F}$. To see it satisfies B1, let $b \in P$ and $\theta(b) I c$. Then $a I^{2} b I c$, so $a I^{3} c$ and hence $c \in L$ with $b I^{\prime} c$. As $\theta(c)=c$, this proves B1. For B2, let $c \in L$ and $b I \theta(c)$. Then $a I^{3} c$ and $b I c$, hence $c I b$ by symmetry, so $a I^{4} b$. But then $a I^{2} b$ by $\mathrm{O} 1$, so $b \in P$, with $b I^{\prime} c$ and $\theta(b)=b$.

We have now shown that $\theta$ is a surjective bounded morphism from $\mathscr{F}^{\prime}$ onto $\mathscr{F}$. It remains to show that $\mathscr{F}^{\prime}$ is a a quasi-2-plane. For Q1, take two points $b, d \in P$. Then $b I^{2} d$ as they are in the same equivalence class, so bIcId for some $d$. As $b \in P$ and $b I c$ we get $c \in L$ as above, so $c$ is a line. As $b I c$ and $d I c$, this line passes through $b$ and $d$. The proof of $\mathrm{Q} 2$ is similar: any two lines $b, d \in L$ have $b I c I d$, for some $c \in P$ with $c$ lying on $b$ and $d$.

That concludes the first case. The alternative is that $\mathscr{F}$ has only one $I^{2}$ equivalence class. Then $a I^{2} b$ for all $a, b \in X$. Take two disjoint copies $P_{0}=\left\{a_{0}\right.$ : $a \in X\}$ and $L_{1}=\left\{a_{1}: a \in X\right\}$ of $X$ and let $\mathscr{F}^{\prime}=\left(P_{0}, L_{1}, I^{\prime}\right)$, where $a_{0} I^{\prime} b_{1}$ iff $a I b$. Let $\theta: P_{0} \cup L_{1} \rightarrow X$ be the projection: $\theta\left(a_{0}\right)=\theta\left(a_{1}\right)=a$.

The definition of $I^{\prime}$ ensures that $\theta$ is a homomorphism from $\mathscr{F}^{\prime}$ onto $\mathscr{F}$. For $\mathrm{B} 1$, if $a_{0} \in P_{0}$ and $\theta\left(a_{0}\right) I b$, then $a I b$ and so $a_{0} I^{\prime} b_{1}$ with $\theta\left(b_{1}\right)=b$. The proof of B2 is similar. Thus $\theta$ is a bounded morphism.

It remains to show that $\mathscr{F}^{\prime}$ is a a quasi-2-plane in this case. But given points $a_{0}, b_{0} \in P_{0}$ in $\mathscr{F}^{\prime}$ we have $a I^{2} b$ in $\mathscr{F}$, so there exists $c$ with $a I c I b$, hence $b I c$, so that $a_{0} I^{\prime} c_{1}$ and $b_{0} I^{\prime} c_{1}$, making $c_{1}$ a line in $\mathscr{F}^{\prime}$ that passes through $a_{0}$ and $b_{0}$. That proves Q1. The proof of $\mathrm{Q} 2$ is similar: any two lines $a_{1}, b_{1}$ of $\mathscr{F}^{\prime}$ have $a I c I b$ for some $c$, and $c_{0}$ is a point in $\mathscr{F}^{\prime}$ lying on both lines.

Corollary 6. Every connected quasi-1-plane is a bounded morphic image of a non-degenerate projective 1-plane of the form $\mathscr{G}_{+}$where $\mathscr{G}$ is a projective 2-plane.

Proof. Let $\mathscr{F}=(X, I)$ be a connected quasi-1-plane. By the Theorem there is a quasi-2-plane $\mathscr{F}^{\prime}$ and a surjective bounded morphism from $\mathscr{F}^{\prime}$ to $\mathscr{F}$. By Theorem 1 there is a projective 2-plane $\mathscr{G}$ and a surjective bounded morphism from $\mathscr{G}$ to $\mathscr{F}^{\prime}$. By Lemma 4(1) these compose to give a surjective bounded morphism $\mathscr{G} \rightarrow \mathscr{F}$, which by Lemma 4(2) also acts as a surjective bounded morphism $\mathscr{G}_{+} \rightarrow \mathscr{F}$ of 1 -frames. By Theorem 2, $\mathscr{G}_{+}$is a non-degenerate projective 1-plane. 


\section{The elliptic case}

A quasi-1-plane is elliptic when it has only one $I^{2}$-equivalence class, a property expressed simply by

O5: For all $a$ and $b, a I^{2} b$.

This condition implies $\mathrm{O} 1$ and $\mathrm{O} 2$ (equivalently connectedness) as well as seriality of $I$, so we can characterise an elliptic quasi-1-plane as a 1-frame satisfying 05 and symmetry, and an elliptic 1-plane as a 1-frame satisfying $\mathrm{O} 5$ and $\mathrm{O} 3$.

Lemma 7. Any 1-frame satisfying $O 5$ and $O 3$ is symmetric.

Proof. Let $a I b$. By seriality there exists $x$ with $b I x$. By O5 $x I^{2} a$, so there is a $y$ with $x I y I a$. Now we have a 4-cycle aIbIxIyIa. By O4 either $a=x$ or $b=y$. In either case $b I a$.

Pledger suggested that the theory based on $\mathrm{O} 5$ and $\mathrm{O} 3$ "can be regarded as elliptic plane geometry in a general sense which permits degenerate planes" [14, p.18]. He gave an analysis of degenerate elliptic planes, showing that they can be excluded by the following shorter substitute for O4 (see [14, (79)]):

O4': There exist $a, b, c, d$ such that $a I b I c$ and $a \neq c$ and $b \neq d$, and neither $a I c$ nor $b I d$.

The elliptic quasi-1-plane depicted on the right of Figure 2 satisfies O4'.

The projective 1-plane $\mathscr{F}_{0}$ depicted in Figure 3 has four vertices, with an edge

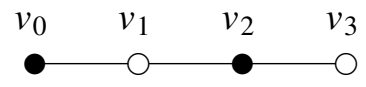

Figure 3: $\mathscr{F}_{0}$

joining $v_{i}$ to $v_{i+1}$ for each $i<3$, and no other edges. It satisfies $\mathrm{O}^{\prime}$. Any given symmetric frame will satisfy $\mathrm{O}^{\prime}$ ' if it contains (a copy of) $\mathscr{F}_{0}$ as a full subgraph, i.e. the frame has no edges between vertices of $\mathscr{F}_{0}$ other than the three edges already belonging to $\mathscr{F}_{0}$.

We want to extend Corollary 6 to the elliptic case, to show that any elliptic quasi1-plane is a bounded morphic image of an elliptic 1-plane that is non-degenerate in the sense of $\mathrm{O}^{\prime}$. But the first step in the proof of that corollary destroys the 
elliptic property O5. So instead we will directly build a 1-plane out of an elliptic quasi-1-plane by adapting the step by step method of [17].

The uniqueness property $\mathrm{O} 3$ can be given a more evocative formulation when $I$ is symmetric. If $a I b$ we may say that $a$ and $b$ are neighbours. If $a I b I c$ then $b$ is a common neighbour of $a$ and $c$. If also $d$ is a common neighbour of $a$ and $c$, then (using symmetry) we have the 4-cycle aIbIcIdIa, so if $a \neq c$, then $\mathrm{O} 3$ enforces $b=d$. We see that in the presence of symmetry, $\mathrm{O} 3$ is equivalent to the requirement that any two distinct elements have at most one common neighbour. Combining that with $\mathrm{O} 5$ we get that in an elliptic 1-plane any two distinct elements have exactly one common neighbour.

Theorem 8. Every elliptic quasi-1-plane is a bounded morphic image of a nondegenerate elliptic 1-plane.

Proof. This will occupy the rest of the current section. Fix an elliptic quasi-1-plane $\mathscr{F}^{\prime}=\left(X^{\prime}, I^{\prime}\right)$. Define a network to be a structure $\mathcal{N}=(X, I, \theta)$ comprising a symmetric 1 -frame $(X, I)$, which we may denote $\mathscr{F}_{\mathcal{N}}$, and a function $\theta: X \rightarrow X^{\prime}$, which we may denote $\theta_{\mathcal{N}}$. We say that another network $\mathscr{N}^{+}=\left(X^{+}, I^{+}, \theta^{+}\right)$extends $\mathcal{N}$ if $X \subseteq X^{+}, I$ is the restriction of $I^{+}$to $X \times X$, and $\theta$ is the restriction of $\theta^{+}$to $X$.

A network $\mathcal{N}$ is called coherent if it satisfies the following conditions

$\mathrm{C} 1: \theta_{\mathscr{N}}: \mathscr{F}_{\mathcal{N}} \rightarrow \mathscr{F}^{\prime}$ is a homomorphism, i.e. satisfies the forth condition F.

C2: distinct elements of $X$ have at most one common neighbour.

C3: $\mathscr{F}_{0}$ is a full subgraph of $\mathscr{F}_{\mathcal{N}}$.

For instance, we obtain a coherent network $\mathcal{N}_{0}=\left(\mathscr{F}_{0}, \theta_{0}\right)$ by noting that since $\mathscr{F}^{\prime}$ is serial it contains a 3-path $a_{0} I a_{1} I a_{2} I a_{3}$, and putting $\theta_{0}\left(v_{i}\right)=a_{i}$ for all $i \leq 3$. We want to build $\mathcal{N}_{0}$ up to a coherent network $\mathscr{N}$ that satisfies O5 and has $\theta_{\mathcal{N}}$ as a bounded morphism. A given network may fail one or both of these requirements, and instances of such failure are called defects. These will require repair. There are two types of possible defect for a network $\mathcal{N}=(X, I, \theta)$ :

B1-defect: this is a pair $\left(a, b^{\prime}\right) \in X \times X^{\prime}$ such that $\theta(a) I^{\prime} b^{\prime}$ but there is no $b \in X$ with $a I b$ and $\theta(b)=b^{\prime}$.

O5-defect: a pair $(a, b) \in X \times X$ with no common neighbour, i.e. $a I^{2} b$ fails.

We show that any defect in a coherent network can be repaired by constructing a coherent extension that contains the element described as lacking by the defect. Once a defect is repaired, it remains so in all further coherent extensions. 
To repair a B1-defect $\left(a, b^{\prime}\right)$ in a coherent network $\mathcal{N}$, take a new object $b$ not in $X$ or $X^{\prime}$ and extend $\mathcal{N}$ to $\mathcal{N}^{+}$by putting

$$
\begin{aligned}
X^{+} & =X \cup\{b\}, \\
I^{+} & =I \cup\{(a, b),(b, a)\} \\
\theta^{+} & =\theta \cup\left\{\left(b, b^{\prime}\right)\right\} .
\end{aligned}
$$

The definition of $I^{+}$ensures that it is symmetric because $I$ is. Thus $\mathcal{N}^{+}$is a network having $a I^{+} b$ and $\theta^{+}(b)=b^{\prime}$, so $\left(a, b^{\prime}\right)$ is no longer a B1-defect in $\mathscr{N}^{+}$. It remains to check that $\mathcal{N}^{+}$is coherent. For $\mathrm{C} 1$, since $\mathcal{N}$ satisfies $\mathrm{C} 1$ we only have to check that $\theta^{+}(a) I^{\prime} \theta^{+}(b)$ and $\theta^{+}(b) I^{\prime} \theta^{+}(a)$. But this follows because $\theta^{+}(a)=\theta(a) I^{\prime} b^{\prime}=\theta^{+}(b)$ and $I^{\prime}$ is symmetric. For $\mathrm{C} 2$, take two distinct elements of $X^{+}$. If one of them is $b$, then they have at most one common $\mathcal{N}^{+}$-neighbour because $b$ has only the one $\mathcal{N}^{+}$-neighbour $a$. If neither of them is $b$ then they have at most one common neighbour in $\mathcal{N}$ by $\mathrm{C} 2$ for $\mathcal{N}$, and they do not have $b$ as a common neighbour as $b$ has only one neighbour, so they have at most one neighbour in $\mathscr{N}^{+}$. For $\mathrm{C} 3, \mathscr{F}_{0}$ has no extra edges in $\mathcal{N}$ by $\mathrm{C} 3$ for $\mathcal{N}$, and $\mathcal{N}^{+}$has only one more edge, with a vertex $b$ not in $\mathscr{N}$, hence not in $\mathscr{F}_{0}$, so $\mathscr{F}_{0}$ has no extra edges in $\mathcal{N}^{+}$. Thus $\mathcal{N}^{+}$is coherent.

To repair an O5-defect $(a, b)$ in a coherent network $\mathcal{N}$, observe that as $\mathscr{F}^{\prime}$ is elliptic we have $\theta(a)\left(I^{\prime}\right)^{2} \theta(b)$, so there exists a $d \in X^{\prime}$ with $\theta(a) I^{\prime} d I^{\prime} \theta(b)$. Take a new object $c \notin X \cup X^{\prime}$ and extend $\mathcal{N}$ to $\mathscr{N}^{+}$by putting

$$
\begin{aligned}
X^{+} & =X \cup\{c\}, \\
I^{+} & =I \cup\{(a, c),(c, a),(c, b),(b, c)\} \\
\theta^{+} & =\theta \cup\{(c, d)\} .
\end{aligned}
$$

Again the definition of $I^{+}$ensures that it is symmetric because $I$ is. Thus $\mathscr{N}^{+}$is a network having $a I^{+} c I^{+} b$, hence $a\left(I^{+}\right)^{2} b$, so $(a, b)$ is no longer an O5-defect in $\mathscr{N}^{+}$. We check that $\mathcal{N}^{+}$is coherent. For $\mathrm{C} 1$, it suffices since $\mathcal{N}$ satisfies $\mathrm{C} 1$ to show that $\theta^{+}(a) I^{\prime} \theta^{+}(c), \theta^{+}(c) I^{\prime} \theta^{+}(b)$ and their inverses. But this follows because $\theta^{+}(a)=\theta(a) I^{\prime} d=\theta^{+}(c)$ and similarly $\theta^{+}(c)=d I^{\prime} \theta^{+}(b)$, and $I^{\prime}$ is symmetric. For $\mathrm{C} 2$, let $x$ and $y$ be distinct members of $X^{+}$. If one of them, say $y$, is equal to $c$, then $x \in X$. If $x$ and $y=c$ have more than one common neighbour in $\mathscr{N}^{+}$, then their common neighbours must be $a$ and $b$, as those are all the neighbours $c$ has. Hence $a$ and $b$ are neighbours of $x$. But then $a I x I b$, contradicting the fact that $(a, b)$ is an O5-defect. So in this case $x$ and $y$ have at most one common neighbour in $\mathscr{N}^{+}$. The alternative case is that $x$ and $y$ both belong to $X$. Now if $x$ and $y$ both belong to $\{a, b\}$, then they have no common neighbour in $\mathcal{N}$, as $(a, b)$ is an O5-defect, so by construction they have $c$ as their only common neighbour in $\mathcal{N}^{+}$. But if one 
of them, say $x$, does not belong to $\{a, b\}$, then it does not have $c$ as a neighbour, so does not have $c$ as a common neighbour with $y$. Since $x$ and $y$ have at most one common neighbour in $\mathcal{N}$, as $\mathcal{N}$ satisfies $\mathrm{C} 2$, it follows in this case that they continue to have at most one common neighbour in $\mathcal{N}^{+}$. That completes the proof that $\mathrm{C} 2$ holds in $\mathcal{N}^{+}$. Finally, for $\mathrm{C} 3$ we have that $\mathscr{F}_{0}$ has no extra edges in $\mathcal{N}$ by $\mathrm{C} 3$ for $\mathcal{N}$, and $\mathcal{N}^{+}$has only two additional edges both with a vertex $c$ not in $\mathscr{F}_{0}$, so $\mathscr{F}_{0}$ has no extra edges in $\mathcal{N}^{+}$.

We have now shown that any defect in a coherent network is repaired in some coherent extension. By iterating this construction sufficiently we will eventually eliminate all defects. The first step is

Lemma 9. Every coherent network $\mathcal{N}$ has a coherent extension $\mathcal{N}^{+}$such that every defect of $\mathcal{N}$ is repaired in $\mathscr{N}^{+}$.

Proof. Let $\kappa$ be the cardinal of the set of all defects of $\mathcal{N}$ (which is a subset of $\left.\left(X \times X^{\prime}\right) \cup(X \times X)\right)$, and let $\left\langle\delta_{\mu}: \mu<\kappa\right\rangle$ be an indexing of these defects by the ordinals less than $\kappa$. We inductively construct a sequence $\left\langle\mathcal{N}_{\mu}^{*}: \mu<\kappa\right\rangle$ of coherent extensions of $\mathcal{N}$ such that for all $\xi<\mu, \mathcal{N}_{\mu}^{*}$ extends $\mathcal{N}_{\xi}^{*}$.

Put $\mathcal{N}_{0}^{*}=\mathcal{N}$. For $\mu<\kappa$, assuming inductively that a coherent $\mathcal{N}_{\mu}^{*}$ has been defined, let $\mathcal{N}_{\mu+1}^{*}$ be a coherent network extending $\mathcal{N}_{\mu}^{*}$ in which $\delta_{\mu}$ is no longer a defect, as exists by the above constructions. If $\mu$ is a limit ordinal, assuming inductively that the sequence $\left\langle\mathcal{N}_{\xi}^{*}: \xi<\mu\right\rangle$ of coherent networks has been defined and forms a chain under the extension relation, let

$$
\mathscr{N}_{\mu}^{*}=\bigcup_{\xi<\mu} \mathcal{N}_{\xi}^{*}=\left(\bigcup_{\xi<\mu} X_{\xi}^{*}, \bigcup_{\xi<\mu} I_{\xi}^{*}, \bigcup_{\xi<\mu} \theta_{\xi}^{*}\right)
$$

be the union of the $\mathcal{N}_{\xi}^{*}$ s. It is readily checked that the union of an extension-chain of coherent networks is a coherent network extending each member of the chain. That completes the definition of the $\mathscr{N}_{\mu}^{*}$ s.

Now put $\mathcal{N}^{+}=\bigcup_{\mu<\kappa} \mathcal{N}_{\mu}^{*}$, a coherent network extending $\mathcal{N}_{0}^{*}=\mathcal{N}$. For each $\mu<\kappa$, the defect $\delta_{\mu}$ is repaired in $\mathcal{N}_{\mu+1}^{*}$, and hence is repaired in $\mathcal{N}^{+}$.

Next we use this result to construct a countably infinite chain $\left\langle\mathcal{N}_{n}: n<\omega\right\rangle$ that repairs all defects. $\mathscr{N}_{0}$ is the coherent network based on the frame $\mathscr{F}_{0}$, as defined earlier. Assuming inductively that $\mathcal{N}_{n}$ has been defined as a coherent network, let $\mathcal{N}_{n+1}$ be the coherent extension $\mathcal{N}_{n}^{+}$of $\mathcal{N}_{n}$ given by Lemma 9

Now put $\mathcal{N}_{\omega}=\bigcup_{n<\omega} \mathcal{N}_{n}$. $\mathcal{N}_{\omega}$ is coherent, and has no defects, since any purported defect of $\mathcal{N}_{\omega}$ would be a defect of $\mathcal{N}_{n}$ for some $n<\omega$, and hence be repaired in $\mathcal{N}_{n+1}$, therefore not a defect in $\mathcal{N}_{\omega}$ after all.

Let $\mathcal{N}_{\omega}=\left(\mathscr{F}_{\omega}, \theta_{\omega}\right)$. Since $\mathcal{N}_{\omega}$ has no O5-defects, $\mathscr{F}_{\omega}$ satisfies O5. Since $\mathcal{N}_{\omega}$ is coherent it satisfies $\mathrm{C} 2$ (distinct elements have at most one common neighbour) 
and hence $\mathscr{F}_{\omega}$ satsifes O3. Thus $\mathscr{F}_{\omega}$ is an elliptic 1-plane. By $\mathrm{C} 3$ for $\mathscr{N}_{\omega}, \mathscr{F}_{0}$ is a full subgraph of $\mathscr{F}_{\omega}$, so $\mathscr{F}_{\omega}$ is non-degenerate. The map $\theta_{\omega}: \mathscr{F}_{\omega} \rightarrow \mathscr{F}^{\prime}$ is a homomorphism by $\mathrm{C} 1$ for $\mathcal{N}_{\omega}$. Since there are no B1-defects it is a bounded morphism.

To complete the proof of Theorem 8 , that $\mathscr{F}^{\prime}$ is a bounded morphic image of a non-degenerate elliptic 1-plane, it remains only to show that $\theta_{\omega}$ is surjective. Let $c^{\prime}$ be any element of $X^{\prime}$. Take any element $a$ of $\mathscr{F}_{\omega}$. Then as $\mathscr{F}^{\prime}$ is elliptic, $\theta_{\omega}(a)\left(I^{\prime}\right)^{2} c^{\prime}$, so there exists $b^{\prime} \in X^{\prime}$ with $\theta_{\omega}(a) I^{\prime} b^{\prime} I^{\prime} c^{\prime}$. Then by B1 for $\theta_{\omega}$ there exists $b$ with $a I_{\omega} b$ and $\theta_{\omega}(b)=b^{\prime}$, so $\theta_{\omega}(b) I^{\prime} c^{\prime}$. By B1 again there then exists $c$ with $b I_{\omega} c$ and $\theta_{\omega}(c)=c^{\prime}$, which establishes that $\theta_{\omega}$ maps onto $\mathscr{F}^{\prime}$.

\section{Some modal metatheory}

We review the background from propositional modal logic that will be needed. This can be found in a number of texts, such as [3, 5, 6].

Modal formulas $\varphi, \psi, \ldots$ are constructed from a countably infinite set Var of propositional variables by the standard Boolean connectives $\neg, \wedge$ and the unary modality $\square$. The other Boolean connectives $\vee, \rightarrow, \leftrightarrow$ are introduced as the usual abbreviations, and the dual modality $\diamond$ is defined to be $\neg \square \neg$. Formulas $\square^{n} \varphi$ are defined by induction on the natural number $n$ by putting $\square^{0} \varphi=\varphi$ and $\square^{n+1} \varphi=\square\left(\square^{n} \varphi\right)$. Formulas $\diamond^{n} \varphi$ are defined likewise by iterating $\diamond$.

A model $\mathscr{M}=(\mathscr{F}, V)$ for this language is given by a 1 -frame $\mathscr{F}=(X, I)$ and a valuation function $V$ that assigns to each variable $p \in \operatorname{Var}$ a subset $V(p)$ of $X$ which may be thought of as the set of points at which $p$ is true. For each $a \in X$, let $I(a)=\{b \in X: a I b\}$ be the set of I-neighbours of $a$. The relation $\mathscr{M}, a \models \varphi$ of a formula $\varphi$ being true (or satisfied) at a in $\mathscr{M}$ is defined by induction on the formation of $\varphi$ as follows:

- $M, a \vDash p$ iff $x \in V(p)$, for $p \in \operatorname{Var}$.

- $\mathscr{M}, a \vDash \neg \varphi$ iff $\mathscr{M}, a \vDash \varphi$ (i.e. $\operatorname{not} \mathscr{M}, a \vDash \varphi$ ).

- $\mathscr{M}, a \mid \varphi \wedge \psi$ iff $\mathscr{M}, a \mid \varphi$ and $\mathscr{M}, a \mid \psi$.

- $\mathscr{M}, a \models \square \varphi$ iff for every $b \in I(a), \mathscr{M}, b \models \varphi$.

Consequently

- $\mathscr{M}, a \models \diamond \varphi$ iff for some $b \in I(a), \mathscr{M}, b \vDash \varphi$.

- $\mathscr{M}, a \models \square^{n} \varphi$ iff for all $b$ such that $a I^{n} b, \mathscr{M}, b=\varphi$. 
- $\mathscr{M}, a \models \diamond^{n} \varphi$ iff for some $b$ such that $a I^{n} b, \mathscr{M}, b=\varphi$.

A formula $\varphi$ is true in model $\mathscr{M}$ when it is true at all elements of $\mathscr{M}$, and satisfiable in $\mathscr{M}$ when it is true at some element of $\mathscr{M}$, i.e. $\neg \varphi$ is not true in $\mathscr{M}$. A set $\Sigma$ of formulas is satisfiable in $\mathscr{M}$ when there is some element of $\mathscr{M}$ at which all members of $\Sigma$ are simultaneously true. $\Sigma$ is satisfiable in a 1-frame if it is satisfiable in some model on that frame.

A formula $\varphi$ is valid in 1 -frame $\mathscr{F}$ if it is true in all models on $\mathscr{F}$, or equivalently if $\neg \varphi$ is not satisfiable in $\mathscr{F} . \varphi$ is valid in a class $\mathscr{C}$ of 1 -frames if it is valid in all members of $\mathscr{C}$.

Given a bounded morphism $\theta: \mathscr{F}^{\prime} \rightarrow \mathscr{F}$ of 1 -frames and a model $\mathscr{M}=(\mathscr{F}, V)$ on $\mathscr{F}$ we obtain a model $\mathscr{M}^{\prime}=\left(\mathscr{F}^{\prime}, V^{\prime}\right)$ on $\mathscr{F}^{\prime}$ by putting $V^{\prime}(p)=\theta^{-1} V(p)=$ $\left\{a \in X^{\prime}: \theta(a) \in V(p)\right\}$ for all $p \in \operatorname{Var}$. Then for any $a \in X^{\prime}$, if $\varphi$ is a variable,

$$
\mathscr{M}^{\prime}, a \vDash \varphi \text { iff } \mathscr{M}, \theta(a) \vDash \varphi .
$$

An induction on formation of formulas then shows that (5.1) holds for every $a$ and every formula $\varphi$ [3, Proposition 2.14].

Theorem 10. If $\mathscr{F}$ is a bounded morphic image of $\mathscr{F}^{\prime}$, and $\Sigma$ is a set of formulas that is satisfiable in $\mathscr{F}$, then $\Sigma$ is satisfiable in $\mathscr{F}^{\prime}$.

Proof. Let $\theta: \mathscr{F}^{\prime} \rightarrow \mathscr{F}$ be a surjective bounded morphism and $\mathscr{M}$ a model on $\mathscr{F}$ such that $\Sigma$ is a satisfiable at some element $b$ in $\mathscr{M}$. Take an element $a$ in $\mathscr{F}^{\prime}$ such that $b=\theta(a)$. Then from (5.1) we get that every member of $\Sigma$ is true at $a$ in $M^{\prime}$.

Note that this result implies that if $\mathscr{F}$ is a bounded morphic image of $\mathscr{F}^{\prime}$, then any formula $\varphi$ that is valid in $\mathscr{F}^{\prime}$ must be valid in $\mathscr{F}$, for if $\neg \varphi$ were satisfiable in $\mathscr{F}$ it would be satisfiable in $\mathscr{F}^{\prime}$.

An inner subframe of a frame $\mathscr{F}=(X, I)$ is any frame $\mathscr{F}^{\prime}=\left(X^{\prime}, I^{\prime}\right)$ for which $X^{\prime} \subseteq X, I^{\prime}$ is the restriction of $I$ to $X^{\prime}$, and $X^{\prime}$ is closed under $I$ in the sense that $I(a) \subseteq X^{\prime}$ for all $a \in X^{\prime}$. This means that $\mathscr{F}^{\prime}$ is a substructure of $\mathscr{F}$ in which all the $I$-neighbours of an element of $\mathscr{F}^{\prime}$ belong to $\mathscr{F}^{\prime}$ as well. It is equivalent to requiring that the inclusion function $X^{\prime} \hookrightarrow X$ is a bounded morphism from $\mathscr{F}^{\prime}$ to $\mathscr{F}$. Thus from any model $\mathscr{M}$ on $\mathscr{F}$ we get a model $\mathscr{M}^{\prime}$ on the inner subframe $\mathscr{F}^{\prime}$ with $V^{\prime}(p)=V(p) \cap X^{\prime}$ such that by (5.1) with $\theta$ as the inclusion,

$$
\mathscr{M}^{\prime}, a \models \varphi \text { iff } \mathscr{M}, a \vDash \varphi \text { for every } a \in X^{\prime} \text { and every formula } \varphi \text {. }
$$

An important case of inner subframe is the notion of the subframe $\mathscr{F}^{a}=\left(X^{a}, I^{a}\right)$ of $\mathscr{F}$ generated by an element $a$. This has

$$
X^{a}=\left\{b \in X: a I^{n} b \text { for some } n \geq 0\right\},
$$

Australasian Journal of Logic (18:4) 2021, Article no. 1 
with $I^{a}$ being the restriction of $I$ to to $X^{a} . \mathscr{F}^{a}$ is often called point-generated. It is the smallest inner subframe of $\mathscr{F}$ that contains $a$ (when $n=0$ ). Note when $b \in X^{a}$ and $b I^{n} c$, then every member of the associated $I$-path of length $n$ from $b$ to $c$ is in $X^{a}$, hence $b\left(I^{a}\right)^{n} c$. For any $b, c \in X^{a}$ we have $a I^{n} b$ and $a I^{m} c$ for some $n, m$, hence $b I_{+}^{n} a I_{+}^{m} c$, showing that there is an $I_{+}^{a}$-path from $b$ to $c$ in $\mathscr{F}^{a}$. Thus

$$
\text { any point-generated frame is connected, }
$$

a fact we will make use of in the next section.

We turn now to matters of proof theory. A normal logic is any set $\Lambda$ of formulas that includes all instances of truth-functional tautologies and of the scheme

$\mathrm{K}: \square(\varphi \rightarrow \psi) \rightarrow(\square \varphi \rightarrow \square \psi)$,

and is closed under the following rules:

modus ponens: if $\varphi, \varphi \rightarrow \psi \in \Lambda$, then $\psi \in \Lambda$;

$\square$-generalisation: if $\varphi \in \Lambda$, then $\square \varphi \in \Lambda$.

The members of a logic $\Lambda$ are called its theorems, or more specifically $\Lambda$-theorems. A formula $\varphi$ is $\Lambda$-consistent when $\neg \varphi$ is not an $\Lambda$-theorem. A set $\Sigma$ of formulas is called $\Lambda$-consistent if any finite conjunction $\varphi_{0} \wedge \cdots \wedge \varphi_{n-1}$ of members of $\Sigma$ is $\Lambda$-consistent. $\Lambda$ is sound for (validity in) a class $\mathscr{C}$ if every $\Lambda$-theorem is valid in $\mathscr{C} . \Lambda$ is complete for (validity in) $\mathscr{C}$ if, conversely, every formula that is valid in $\mathscr{C}$ is a $\Lambda$-theorem. This is equivalent to requiring that every $\Lambda$-consistent formula is satisfiable in some member of $\mathscr{C} . \Lambda$ is strongly complete for $\mathscr{C}$ if every $\Lambda$-consistent set of formulas is satisfiable in some member of $\mathscr{C}$.

Any instance of the formula scheme $\mathrm{K}$ is valid in any 1-frame, and this validity is preserved by the modus ponens and $\square$-generalisation rules. Thus for any $\mathscr{F}$, the set of all formulas valid in $\mathscr{F}$ is a normal logic. The smallest (intersection) of all normal logics, also known as $\mathrm{K}$, is sound and strongly complete for validity in the class of all 1-frames. One way to prove that is to use the canonical frame $\mathscr{F}_{\Lambda}=\left(X_{\Lambda}, I_{\Lambda}\right)$, which can be defined for any $\Lambda$ by taking $X_{\Lambda}$ to be the set of all maximally $\Lambda$-consistent sets of formulas, with $a I_{\Lambda} b$ when $\{\varphi: \square \varphi \in a\} \subseteq b$. The canonical model $\mathscr{M}_{\Lambda}=\left(\mathscr{F}_{\Lambda}, V_{\Lambda}\right)$ has $V_{\Lambda}(p)=\left\{a \in X_{\Lambda}: p \in a\right\}$ for each variable $p$. It has the property that any formula $\varphi$ is true at an element $a \in X_{\Lambda}$ iff $\varphi$ belongs to the maximally $\Lambda$-consistent set $a$, i.e.

$$
\mathscr{M}_{\Lambda}, a \vDash \varphi \text { iff } \varphi \in a .
$$

This is sometimes called the Truth Lemma for $\Lambda$ : for details see [3, Chapter 4] or [6. Chapter 3]. Since a formula $\varphi$ is a $\Lambda$-theorem iff it belongs to every member 
of $X_{\Lambda}$, the Truth Lemma implies that the formulas that are true in the model $M_{\Lambda}$ are precisely the $\Lambda$-theorems. Consequently, any formula that is valid in the frame $\mathscr{F}_{\Lambda}$ must be a $\Lambda$-theorem. Equivalently, every $\Lambda$-consistent formula is satisfiable in $\mathscr{M}_{\Lambda}$, hence satisfiable in $\mathscr{F}_{\Lambda}$. More strongly, any $\Lambda$-consistent set $\Sigma$ of formulas can be extended to a maximally $\Lambda$-consistent set $a_{\Sigma}$, and so by the Truth Lemma $\Sigma$ is satisfied at $a_{\Sigma}$ in $\mathscr{M}_{\Lambda}$, so is satisfiable in $\mathscr{F}_{\Lambda}$.

Now letting $\Lambda=\mathrm{K}$, we get the strong completeness of the logic K: every Kconsistent set of formulas is satisfiable in some 1-frame, namely in the model $\mathscr{M}_{\mathrm{K}}$ on the frame $\mathscr{F}_{\mathrm{K}}$.

\section{Strong completeness of $12 \mathrm{~g}$ and $8 f$}

The canonical frame construction provides a method for showing that a logic $\Lambda$ is strongly complete for a class $\mathscr{C}$. Since every $\Lambda$-consistent set of formulas is satisfiable in $\mathscr{F}_{\Lambda}$, it is enough to show that $\mathscr{F}_{\Lambda}$ belongs to $\mathscr{C}$. If $\mathscr{C}$ is defined by specified properties, then the strong completeness proof is reduced to showing that $\mathscr{F}_{\Lambda}$ has those properties. There are numerous logics for which the axioms defining $\Lambda$ can be directly applied to show that $\mathscr{F}_{\Lambda}$ has the properties in question.

The first very general result of this kind appeared in [10, Section 4], which defined the axiom scheme

$$
\mathrm{G}^{\prime}: \diamond^{m} \square^{n} \varphi \rightarrow \square^{p} \diamond^{q} \varphi
$$

The parameters $m, n, p, q$ are a fixed but arbitrary quadruple of natural numbers. Corresponding to $\mathrm{G}^{\prime}$ is the frame condition

$\left(\mathrm{g}^{\prime}\right)$ : for all $a, b, c$ such that $a I^{m} b$ and $a I^{p} c$, there exists $d$ with $b I^{n} d$ and $c I^{q} d$.

It is proved in Theorems 4.1 and 4.2 of [10] that

- $\mathrm{G}^{\prime}$ is valid in any 1 -frame satisfying $\left(\mathrm{g}^{\prime}\right)$.

- If a normal logic $\Lambda$ includes $\mathrm{G}^{\prime}$, then $\mathscr{F}_{\Lambda}$ satisfies $\left(\mathrm{g}^{\prime}\right)$.

The converse of the first item is also true, so in fact a 1-frame validates $\mathrm{G}^{\prime}$ if, and only if, it satisfies $\left(\mathrm{g}^{\prime}\right)$.

Many interesting cases can be read off from this analysis. Table 1 gives the four that we use. The left column gives particular values of the parameters of $\mathrm{G}^{\prime}$, the middle column gives the resulting instance of $\mathrm{G}^{\prime}$ with a name, and the third column gives the corresponding condition on $I$ expressed by $\left(\mathrm{g}^{\prime}\right)$.

The names D and B are standard. The name $4^{2}$ is by analogy with the standard name 4 for the axiom $\square \varphi \rightarrow \square^{2} \varphi$ which corresponds to $I$ being transitive. 


\begin{tabular}{l|l|l}
$m, n, p, q$ & \multicolumn{1}{|c|}{$\mathrm{G}^{\prime}$} & \multicolumn{1}{|c}{$\mathrm{g}^{\prime}$} \\
\hline $0,1,0,1$ & $\mathrm{D}: \square \varphi \rightarrow \diamond \varphi$ & $I$ is serial \\
$1,1,0,0$ & $\mathrm{~B}: \diamond \square \varphi \rightarrow \varphi$ & $I$ is symmetric \\
$0,2,4,0$ & $4^{2}: \square^{2} \varphi \rightarrow \square^{4} \varphi$ & $a I^{4} b$ implies $a I^{2} b$, i.e. O1 \\
$0,3,0,0$ & $\mathrm{~T}^{3}: \square^{3} \varphi \rightarrow \varphi$ & $a I^{3} a$, i.e. $I^{3}$ is reflexive
\end{tabular}

Table 1: Cases of $\mathrm{G}^{\prime}$

$4^{2}$ corresponds to $I^{2}$ being transitive. $\mathrm{T}^{3}$ is by analogy with the name $\mathrm{T}$ which corresponds to $I$ being reflexive.

$12 \mathrm{~g}$ can now be defined as the smallest normal logic that includes the axiom schemes D, B and $4^{2}$. By the quoted results from [10], a 1-frame validates these three axioms iff it is serial, symmetric and satisfies O1, i.e. iff it is a quasi-1-plane. The set of all formulas valid in a given quasi-1-plane is a normal logic including $\mathrm{D}, \mathrm{B}$ and $4^{2}$, so it includes $12 \mathrm{~g}$ as the smallest such logic. Thus $12 \mathrm{~g}$ is sound for validity in the class of all quasi-1-planes. Moreover the canonical frame $\mathscr{F}_{12 \mathrm{~g}}$ for $12 \mathrm{~g}$ is a quasi-1-plane, so $12 \mathrm{~g}$ is strongly complete for quasi-1-planes. This can be combined with our Corollary 6 to yield the stronger

Theorem 11. $12 \mathrm{~g}$ is strongly complete for the class of all non-degenerate projective 1-planes.

Proof. Let $\Sigma$ be a $12 \mathrm{~g}$-consistent set of formulas. Then $\Sigma$ is satisfied in the canonical model $\mathscr{M}_{12 \mathrm{~g}}$ at some element $a$. Let $\mathscr{F}$ be the inner subframe of $\mathscr{F}_{12 \mathrm{~g}}$ generated by $a$. The properties of being serial, symmetric and satisfying $\mathrm{O} 1$ are all preserved in passing from $\mathscr{F}_{12 \mathrm{~g}}$ to $\mathscr{F}$, so $\mathscr{F}$ is a quasi-1-plane. Moreover, being point-generated it is connected by (5.3). Therefore by Corollary 6 there exists a non-degenerate projective 1-plane $\mathscr{F}^{*}$ with a surjective bounded morphism $\theta: \mathscr{F}^{*} \rightarrow \mathscr{F}$.

Now the model $\mathscr{M}_{12 \mathrm{~g}}$ on $\mathscr{F}_{12 \mathrm{~g}}$ restricts to a model $\mathscr{M}$ on $\mathscr{F}$ in which $\Sigma$ is satisfied at $a($ see $(5.2)$ ). So $\Sigma$ is satisfiable in $\mathscr{F}$. Hence by Theorem $10, \Sigma$ is satisfiable in $\mathscr{F}^{*}$ (at some point $a^{\prime}$ with $\theta\left(a^{\prime}\right)=a$ ).

This shows that every $12 \mathrm{~g}$-consistent set of formulas is satisfiable in some non-degenerate projective 1-plane, as required.

This result is a manifestation of the fact that the modal language does not have the expressive power to differentiate the non-degenerate projective 1-planes from other 1-planes, including the elliptic ones, or even from the other quasi-planes:

Theorem 12. For any formula $\varphi$, the following are equivalent. 
(1) $\varphi$ is a theorem of $12 g$.

(2) $\varphi$ is valid in all quasi-1-planes.

(3) $\varphi$ is valid in all connected quasi-1-planes.

(4) $\varphi$ is valid in all 1-planes.

(5) $\varphi$ is valid in all projective-1-planes.

(6) $\varphi$ is valid in all non-degenerate projective-1-planes.

Proof. That 1 implies 2 is the soundness of $12 \mathrm{~g}$ shown above. That 2 implies 3, 3 implies 4, 4 implies 5 and 5 implies 6 are all immediate, since the antecedent class includes the consequent one. That 6 implies 1 follows from the completeness of $12 \mathrm{~g}$ for non-degenerate projective-1-planes given by Theorem 11 .

This result tell us that if a formula is valid in all projective 1-planes (5), then it is valid in all 1-planes (4), and in particular is valid in all the elliptic ones. On the other hand there are formulas that are valid in all elliptic 1-planes but not in all projective ones. Note first that the elliptic condition O5 is not itself equivalent to the validity of any modal formula. This is readily seen from the fact that the class of 1-frames validating a modal formula is closed under disjoint unions [3, Theorem 3.14], but the disjoint union of two frames satisfying O5 will not satisfy O5. Nonetheless the modal language can differentiate elliptic structures. For 1planes a suitable condition is that $a I^{3} b$ implies $a I^{2} b$, which is equivalent to the validity of the scheme $\square^{2} \varphi \rightarrow \square^{3} \varphi$. In the presence of $\mathrm{O} 2$ this yields O5, hence ensures there is one $I^{2}$-equivalence class [14, p.17]. But there is a slightly simpler condition that does not depend on $\mathrm{O} 2$ :

Lemma 13. A connected quasi-1-plane $\mathscr{F}=(X, I)$ is elliptic iff $I^{3}$ is reflexive iff there exists an element a with a $I^{3} a$.

Proof. By Theorem 3 (4), for any $a \in X$ the subsets $I^{2}(a)$ and $I^{3}(a)$ are the only $I^{2}$ equivalence classes. If $\mathscr{F}$ is elliptic then for any $a, I^{2}(a)=I^{3}(a)$, so $a I^{3} a$ because $a I^{2} a$. But for the converse it only takes one $a$ to have $a I^{3} a$ to get $a \in I^{2}(a) \cap I^{3}(a)$ and hence the equivalence classes are equal.

The logic $8 \mathrm{f}$ can be defined as the smallest extension of $12 \mathrm{~g}$ that includes the axiom scheme $\mathrm{T}^{3}$. That scheme corresponds to reflexivity of $I^{3}$ (Table 1 ). Thus $8 \mathrm{f}$ is sound for validity in the class of all elliptic quasi-1-planes [14, pp.33, 40].

Theorem 14. $8 f$ is strongly complete for the class of all non-degenerate elliptic 1-planes. 
Proof. Let $\Sigma$ be an $8 \mathrm{f}$-consistent set of formulas. Then by the argument of the proof of Theorem 11, $\Sigma$ is satisfied at some element $a$ in the inner subframe $\mathscr{F}$ of the canonical frame $\mathscr{F}_{8 \mathrm{f}}$ generated by $a$, and $\mathscr{F}$ is a connected quasi-1-plane. But now by [10,4.2], since $\mathrm{T}^{3}$ is included in $8 \mathrm{f}, \mathscr{F}_{8 \mathrm{f}}$ has $I^{3}$ reflexive. That property is preserved in passing to $\mathscr{F}$, since if $b I_{8 \mathrm{f}} c I_{8 \mathrm{f}} d I_{8 \mathrm{f}} b$ and $b$ is in $\mathscr{F}$, then $c$ and $d$ are in $\mathscr{F}$ with bIcIdIb. Thus $\mathscr{F}$ is an elliptic quasi-1-plane by Lemma 13 . Hence by Theorem 8 , there exists a non-degenerate elliptic 1-plane $\mathscr{F}^{*}$ with a surjective bounded morphism $\theta: \mathscr{F}^{*} \rightarrow \mathscr{F}$. So by Theorem $10, \Sigma$ is satisfiable in $\mathscr{F}^{*}$.

Another property of a relation $I$ that it is natural to consider is irreflexiveness, which is well known not to be equivalent to the validity of any modal formula (e.g. it is not preserved by surjective bounded morphisms). For a 2-frame $\mathscr{F}$, irreflexiveness is built in since $I \subseteq P \times L$ and $P \cap L=\emptyset$, so aIa never occurs. Hence the associated symmetric 1 -frame $\mathscr{F}_{+}$is irreflexive, and indeed never has $a\left(I_{+}\right)^{n} a$ if $n$ is odd. Thus in applying Corollary 6 to the proof of Theorem 11 . what is produced is an irreflexive 1-plane satisfying a $12 \mathrm{~g}$-consistent set. So $12 \mathrm{~g}$ is strongly complete for the class of irreflexive non-degenerate elliptic 1-planes.

In the real elliptic plane, the conjugacy relation is irreflexive, since no point lies on its polar line, so $a I a$ cannot hold. Irreflexivity of $I$ is one of the axioms for elliptic plane geometry of Kordos [8]. Now in the proof of Theorem 8, the graph $\mathscr{F}_{0}$ is irreflexive and the operations for repairing defects do not introduce any reflexive points, so the non-degenerate elliptic 1-plane constructed in that theorem is irreflexive. It follows by the proof of Theorem 14 , that $8 \mathrm{f}$ is strongly complete for the class of all irreflexive non-degenerate elliptic 1-planes.

\section{$7 \quad$ Finite model property}

A modal logic $\Lambda$ has the finite model property when every $\Lambda$-consistent formula is satisfiable in some model on a finite frame that validates $\Lambda$. Equivalently this means that $\Lambda$ is complete for validity in the class of all finite frames that validate $\Lambda$ (hence sound and complete for this class).

We will show that the logics $12 \mathrm{~g}$ and $8 \mathrm{f}$ have the finite model property. Since they have finitely many axioms, it follows by standard theory that the property of being a theorem of the logic is algorithmically decidable for each of them. For an explanation of this theory see [3, §6.2] or [5, §16.2].

We use the well known filtration method of reducing a model to a finite one. Let $\Lambda$ be a normal logic extending 12g. Fix a formula $\varphi$ that is $\Lambda$-consistent. Then by the construction in the first part of the proof of Theorem 11, $\varphi$ is true at some element of a model $\mathscr{M}$ on a connected quasi-1-plane $\mathscr{F}=(X, I)$ which is a point-generated subframe of the canonical frame $\mathscr{F}_{\Lambda}$. Let $\Phi$ be the finite set of all subformulas of 
$\varphi$. For each $a \in X$, let $\Phi_{a}=\{\psi \in \Phi: \mathscr{M}, a \vDash \psi\}$. Define an equivalence relation $\sim$ on $X$ by putting $a \sim b$ iff $\Phi_{a}=\Phi_{b}$. Then with $|a|=\{b \in X: a \sim b\}$ being the $\sim$-equivalence class of $a$, we put $X_{\Phi}=\{|a|: a \in X\}$. The set $X_{\Phi}$ is finite, because the map $|a| \mapsto \Phi_{a}$ is a well-defined injection of $X_{\Phi}$ into the powerset of $\Phi$. Thus $X_{\Phi}$ has size at most $2^{n}$, where $n$ is the size of $\Phi$.

A filtration of $\mathscr{M}$ through $\Phi$ is any model $\mathscr{M}^{\prime}=\left(\mathscr{F}^{\prime}, V^{\prime}\right)$ with $\mathscr{F}^{\prime}$ of the form $\left(X_{\Phi}, I^{\prime}\right)$ such that

(i) $a I b$ implies $|a| I^{\prime}|b|$, i.e. $a \mapsto|a|$ is a homomorphism $\mathscr{F} \rightarrow \mathscr{F}^{\prime}$.

(ii) if $|a| I^{\prime}|b|$, then for all $\square \psi \in \Phi, \mathscr{M}, a \models \square \psi$ implies $\mathscr{M}, b=\psi$.

(iii) $V^{\prime}(p)=\left\{|a| \in X_{\Phi}: a \in V(p)\right\}$ for all variables $p \in \Phi$.

For such an $\mathscr{M}^{\prime}$, we have the Filtration Theorem: for any $\psi \in \Phi$ and $a$ in $\mathscr{F}$,

$$
\mathscr{M}, a \mid=\psi \text { iff } \mathscr{M}^{\prime},|a|=\psi .
$$

This is shown by induction on the formation of the formula $\psi \in \Phi$ : see [3] Theorem. 2.39] or [5, Theorem. 5.23].

Filtrations of $\mathscr{M}$ through $\Phi$ do exist. The least filtration has $V^{\prime}(p)$ defined by (iii) if $p \in \Phi$ and $V^{\prime}(p)=\emptyset$ (or anything) otherwise; while $I^{\prime}$ is defined for $\alpha, \beta \in X_{\Phi}$ by putting

$$
\alpha I^{\prime} \beta \text { iff there exist } a \in \alpha \text { and } b \in \beta \text { such that } a I b \text {. }
$$

This definition makes $I^{\prime}$ symmetric whenever $I$ is, and serial whenever $I$ is.

We are now in a position to demonstrate that $8 \mathrm{f}$ has the finite model property. Put $\Lambda=8 \mathrm{~g}$ in the above and let $\mathscr{M}^{\prime}$ be the least filtration of $\mathscr{M}$ through $\Phi$. Now the underlying frame $\mathscr{F}$ of $\mathscr{M}$ is a subframe of $\mathscr{F}_{8 \mathrm{f}}$ that is an elliptic quasi-1-plane by the argument in the proof of Theorem 14 , so $I$ is symmetric and satisfies O5. Hence $I^{\prime}$ is symmetric, as just noted. It also satisfies O5, for if $|a|,|b| \in X_{\Phi}$, then $a I c I b$ for some $c$ as $\mathscr{F}$ satisfies O5, hence $|a| I^{\prime}|c| I^{\prime}|b|$ by the filtration homomorphism property (i). Thus $\mathscr{F}^{\prime}$ is an elliptic quasi-1-plane and therefore validates $8 \mathrm{f}$. But $\varphi$ is true at some point $a$ of $\mathscr{M}$, and $\varphi \in \Phi$, so $\varphi$ is true at $|a|$ in $\mathscr{M}^{\prime}$ by the Filtration Theorem. This proves that any $8 \mathrm{f}$-consistent formula is satisfiable in a model on a finite frame validating $8 \mathrm{f}$, as required.

In the case that $\Lambda=12 \mathrm{~g}$, we only know so far that the subframe $\mathscr{F}$ of $\mathscr{F}_{12 \mathrm{~g}}$ is a connected quasi-1-plane that may be projective or elliptic. If it is elliptic, we can proceed exactly as in the preceding paragraph to obtain a satisfying model for $\varphi$ on a finite elliptic quasi-1-plane. If however $\mathscr{F}$ is projective, we proceed to construct a suitable $\mathscr{M}^{\prime}$ in a different way, with new definitions of $X_{\Phi}$ and $I^{\prime}$ as follows. $\mathscr{F}$ 
has two $I^{2}$-equivalence classes, which we will label $P$ and $L$. We filtrate $P$ and $L$ separately through $\Phi$. For each $a \in P$, let $|a|=\{b \in P: a \sim b\}$, while if $a \in L$ let $|a|=\{b \in L: a \sim b\}$. Put $P^{\prime}=\{|a|: a \in P\}, L^{\prime}=\{|a|: a \in L\}$, and $X_{\Phi}=P^{\prime} \cup L^{\prime}$. Since $P$ and $L$ are disjoint, so too are $P^{\prime}$ and $L^{\prime}$, which each have at most $2^{n}$ elements. Hence $X_{\Phi}$ is of size at most $2^{n+1}$. Then $I^{\prime}$ is defined for $\alpha, \beta \in X_{\Phi}$ by the condition (7.1). To complete the definition of $\mathscr{M}^{\prime}$ we define $V^{\prime}(p)$ by (iii) if $p \in \Phi$, and put $V^{\prime}(p)=\emptyset$ otherwise, as before.

$\mathscr{M}^{\prime}$ is a filtration of $\mathscr{M}$ through $\Phi$ : (iii) holds by definition of $V^{\prime}$, and reading (7.1) from right to left shows that $a I b$ implies $|a| I^{\prime}|b|$, i.e. (i) holds. For (ii), suppose $|a| I^{\prime}|b|$. Then $a^{\prime} I^{\prime} b^{\prime}$ for some $a^{\prime} \in|a|$ and $b^{\prime} \in|b|$, so if $\square \psi \in \Phi$ and $\mathscr{M}, a \mid \square \psi$, then $\mathscr{M}, a^{\prime} \vDash \square \psi$ as $a \sim a^{\prime}$, hence $\mathscr{M}, b^{\prime} \vDash \psi$ as $a^{\prime} I b^{\prime}$, and $\mathscr{M}, b \models \psi$ as $b \sim b^{\prime}$, which proves (ii). It follows that the Filtration Theorem holds for $\mathscr{M}^{\prime}$, leading us to conclude that the $12 \mathrm{~g}$-consistent formula $\varphi$ is satisfiable in the finite model $\mathscr{M}^{\prime}$.

It remains to show that $\mathscr{F}^{\prime}=\left(X_{\Phi}, I^{\prime}\right)$ validates $12 \mathrm{~g}$. As noted above, (7.1) ensures that the properties of being serial and symmetric are preserved in passing from $I$ to $I^{\prime}$. We show that $I^{\prime}$ satisfies O1. First, by parts (4) and (5) of Theorem 3 , whenever $a I b$ then one of $a$ and $b$ belongs to $P$ and the other belongs to $L$. Hence by (7.1), whenever $\alpha I^{\prime} \beta$ then one of $\alpha$ and $\beta$ belongs to $P^{\prime}$ and the other belongs to $L^{\prime}$. Iterated application of this gives that if $\alpha\left(I^{\prime}\right)^{n} \beta$ and $n$ is even, then $\alpha$ and $\beta$ both belong to $P^{\prime}$, or both belong to $L^{\prime}$. We can now prove O1: suppose $\alpha\left(I^{\prime}\right)^{4} \beta$. If $\alpha \in P^{\prime}$, then $\beta \in P^{\prime}$, so choosing $a \in \alpha$ and $b \in \beta$ we have $a, b \in P$, hence $a I^{2} b$ as $P$ is an $I^{2}$-equivalence class. But then $|a|\left(I^{\prime}\right)^{2}|b|$ as in the $8 \mathrm{f}$ case, i.e. $\alpha\left(I^{\prime}\right)^{2} \beta$. But if $\alpha \in L^{\prime}$, then $\beta \in L^{\prime}$ and similarly we infer that $\alpha\left(I^{\prime}\right)^{2} \beta$.

This proves that $\mathscr{F}$ satisfies $\mathrm{O} 1$ and is a quasi-1-frame, hence validates $12 \mathrm{~g}$. (In fact it is projective, with $P^{\prime}$ and $L^{\prime}$ being its only two $\left(I^{\prime}\right)^{2}$-equivalence classes.) That completes the proof of the finite model property for $12 \mathrm{~g}$.

We end with a comment on irreflexiveness and the logic 8f. It was shown at the end of Section 6 that $8 \mathrm{f}$ is (strongly) complete for the class of all irreflexive nondegenerate elliptic 1-planes. However it does not have the finite model property for this class. Pledger noted in [14, p.24] that the class has no finite members at all, by the Friendship Theorem of graph theory. That theorem, due to Erdôs, Rényi and Sós, can be formulated as saying that a finite irreflexive symmetric 1-frame in which any two distinct elements have exactly one common neighbour must be a windmill graph, a set of triangles that all have one common vertex, while no two triangles share any other vertex (see [1, Chapter 44] for an exposition). Thus any finite irreflexive elliptic 1-plane is a windmill. But no windmill can satisfy the non-degeneracy condition $\mathrm{O}^{\prime}$, as the reader may like to confirm. 


\section{Acknowledgement}

My thanks to Shirley Pledger for making [14] available and providing biographical information.

\section{References}

[1] Martin Aigner and Günter M. Ziegler. Proofs From THE BOOK. Springer, sixth edition, 2018.

[2] Philippe Balbiani, Valentin Goranko, Ruaan Kellermanx, and Dimiter Vakarelov. Logical theories for fragments of elementary geometry. In Marco Aiello, Ian Pratt-Hartmann, and Johan van Benthem, editors, Handbook of Spatial Logics, pages 343-428. Springer, 2007.

[3] Patrick Blackburn, Maarten de Rijke, and Yde Venema. Modal Logic. Cambridge University Press, 2001.

[4] George Boolos. The Logic of Provability. Cambridge University Press, 1993.

[5] Alexander Chagrov and Michael Zakharyaschev. Modal Logic. Oxford University Press, 1997.

[6] Robert Goldblatt. Logics of Time and Computation. CSLI Lecture Notes No. 7. CSLI Publications, Stanford University, second edition, 1992.

[7] Ian Hodkinson and Altaf Hussain. The modal logic of affine planes is not finitely axiomatisable. The Journal of Symbolic Logic, 73(3):940-952, 2008.

[8] M. Kordos. Elliptic geometry as a theory of one binary relation. Bull. Acad. Polon. Sci. Sér. Sci. Math. Astronom. Phys., 21:609-614, 1973.

[9] Saul A. Kripke. Semantical analysis of modal logic I. Normal modal propositional calculi. Zeitschrift für Mathematische Logik und Grundlagen der Mathematik, 9:67-96, 1963.

[10] E. J. Lemmon. An Introduction to Modal Logic, volume 11 of American Philosophical Quarterly Monograph Series. Basil Blackwell, Oxford, 1977. Written in 1966 in collaboration with Dana Scott. Edited by Krister Segerberg.

[11] K. E. Pledger. Modalities of systems containing S3. Zeitschrift für Mathematische Logik und Grundlagen der Mathematik, 18:267-283, 1972. 
[12] K. E. Pledger. Some extensions of S3. Notre Dame Journal of Formal Logic, 16(2):271-272, 1975.

[13] K. E. Pledger. Location of some modal systems. Notre Dame Journal of Formal Logic, 21(4):683-684, 1980.

[14] K. E. Pledger. Some Interrelations between geometry and modal logic. $\mathrm{PhD}$ thesis, University of Warsaw, 1980. Reprinted as the second article in this issue of the Australasian Journal of Logic, (18:4) 2021.

[15] Raphael M. Robinson. Binary relations as primitive notions in elementary geometry. In Leon Henkin, Patrick Suppes, and Alfred Tarski, editors, The Axiomatic Method, Studies in Logic, pages 68-85. North-Holland, 1959.

[16] L. W. Szczerba and A. Tarski. Metamathematical discussion of some affine geometries. Fundamenta Mathematicae, 104(3):155-192, 1979.

[17] Yde Venema. Points, lines and diamonds: A two-sorted modal logic for projective planes. Journal of Logic and Computation, 9(5):601-621, 1999. 